\title{
On Identifying Functionally Untestable Transition Faults *
}

\author{
Xiao Liu ${ }^{\dagger}$ and Michael S. Hsiao \\ ${ }^{\dagger}$ Texas Instruments, Dallas, TX \\ ${ }^{\ddagger}$ Dept. of ECE, Virginia Tech, Blacksburg, VA
}

\begin{abstract}
This paper presents a new approach on identifying functionally untestable transition faults in non-scan sequential circuits. We formulate a new dominance relationship for transition faults and use it to identify more sequentially untestable transition faults. The proposed method consists of two phases: first, a large number of functionally untestable transition faults is identified by a fault-independent sequential logic implications implicitly crossing multiple time-frames, and the identified untestable faults are classified into three conflict categories. Next, additional functionally untestable transition faults are identified by dominance relationships from the previous identified untestable transition faults. The experimental results for ISCAS89 sequential benchmark circuits showed that our approach can quickly identify many more functionally untestable transition faults than previously reported.
\end{abstract}

\section{Introduction}

Higher clock rate, shrinking geometries, increasing metal density, etc. introduce various defects that stuck-at tests cannot screen out. Therefore, delay fault testing, which verifies that the CUT operate correctly at desired speed, is becoming a necessity to maintain the product quality level. However, (non-scan) functional delay testing can be impractical for large circuits in that large test sets may be required to achieve a desirable fault coverage. As a result, at-speed $\mathrm{AC}$ scan testing has been widely used in the industry to detect delay-induced defects. Compared to functional testing, scan-based testing for delay faults can decrease the overall ATPG complexity and cost, since both controllability and observability on the flipflops are enhanced. However, some of the functionally untestable faults which do not impair the normal operation of the circuit may become testable in scan testing [1]. This scenario is known as overtesting and may result in yield loss [2]. Unfortunately, identifying the functionally untestable transition faults in large-scale sequential circuit

\footnotetext{
* research conducted at Virginia Tech, and supported in part by NSF grants 0196470, 0305881, and an Intel grant
}

can be prohibitively expensive, because it is of the same complexity as sequential ATPG, which is of exponential complexity in terms of the size of the circuit.

For delay faults, several approaches have been proposed on the identification of functionally untestable delay faults. In [3], a general delay fault model, which allows the delay faults to persist for many cycles, is introduced, and algorithms are developed to identify redundancies of arbitrary size. In [4], a path-independent implication-based approach is proposed for identifying non-robust untestable path delay faults in combinational circuits. Additional general implication-based algorithms are given in [5] on identifying untestable segment delay faults and path delay faults. Most recently, approaches proposed in $[6,7]$ combine the implicationbased approach and ATPG-based technique to identify more untestable transition faults in sequential circuits. It has also been observed that the size of the transition fault (in terms of clock cycles) has to be considered for sequential circuits under at-speed testing [8]. The author in [8] points out that different transition fault sizes will cause different faulty circuit behavior. However, the number of untestable transition faults decreases significantly when the size of the fault increases from one cycle to multiple cycles [6]. Therefore, we will assume the delay defect size is shorter than one clock cycle in this paper.

In this paper, we present a new approach on identifying functionally untestable transition faults in non-scan sequential circuits. The proposed method consists of two phases: first, a large number of functionally untestable transition faults is quickly identified by using a faultindependent logic implications implicitly crossing multiple time-frames and classified into three conflict categories. Then, the technique identifies additional functionally untestable transition faults by finding the dominated fault sets on the previous identified untestable transition faults. The experimental results for ISCAS89 sequential benchmark circuits showed that our approach can identify many more functionally untestable transition faults than the implication-based method and a ATPG-based method previously reported.

The rest of the paper is organized as follows. Section 2 reviews the logic implication and fault dominance, 
which will be used in the rest of paper. Section 3 describes our proposed two-phase approach for identifying functionally untestable transition faults in sequential circuits. Section 4 gives our experimental results on ISCAS89 benchmark circuits and compares it with the implicationbased method and another ATPG-based identification approach. Finally, Section 5 concludes the paper.

\section{Preliminaries}

\subsection{Static Logic Implication}

Static logic implication (also called static learning) is a procedure which performs implications on both value assignment ( 0 and 1$)$ for each node in the circuit. Since the effectiveness of implication-based untestable fault identification highly depends on the completeness of the implications learned, it is critical to have a large set of implications for individual gates in the circuit. A number of techniques have been reported on implication computation. In [9], a 16-value logic algebra and deduction list method was proposed to determine necessary node assignments in ATPG. Transitive closure procedure was used in [10] to identify indirect implication on implication graphs. A complete implication learning algorithm is based on recursive learning [11]; however, the depth of recursion must be kept low to keep the computation time within reasonable bounds. Extended backward implication was introduced in [12] to identify additional nontrivial implications. And more recently, a compact implication graph [13] has been proposed for sequential circuits, which spans multiple time-frames without suffering from memory explosion.

In our work, direct, indirect and extended backward implications are used. Although direct implications can be easily learned, indirect and extended backward implications require repeated application of contrapositive and transitive laws. The discovery of indirect and extended backward implications are nontrivial and can help us in both ATPG process and untestable fault implications.

The following terminology will be used throughout the remainder of this paper:

1. $[a, v, t]$ : assign logic value $v$ to gate $a$ in time-frame $t$, where $v \in 0,1$. When $t=0,[\mathrm{~N}, v, 0]$ is also expressed simply as [N,v].

2. $[a, v] \rightarrow[b, u, t]:$ assignment to logic value $v$ on gate $a$ in the current time frame implies value $u$ assigned to gate $b$ in time frame $t$.

3. $\operatorname{impl}[a, v, t]$ : the implication set of assigning logic value $v$ to gate $a$ in the time-frame $t$.

4. $\mathrm{a} / v$ : the stuck-at- $v$ fault on gate a.
We will use Figure 1 to illustrate the direct implication, indirect implication and extended backward implication. Without loss of generality, we will look at the impl[g,1].

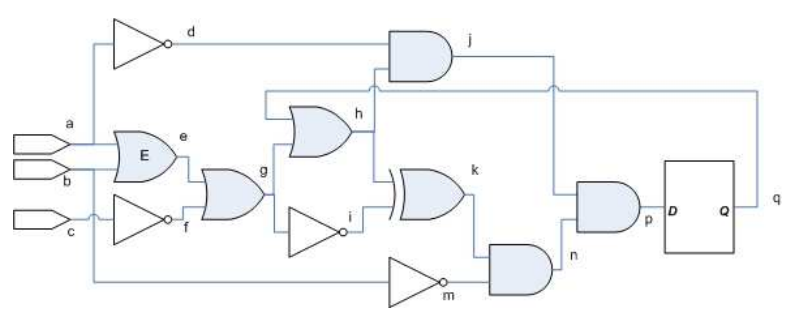

Figure 1: Example sequential circuit

\section{Direct Implication}

This is computed by directly traversing immediate fan-in and fanout of every gate in the circuit. For example, with the immediate fan-in and fanout of gate $g$, we find that $[g, 1] \rightarrow\{[e, 1],[f, 1],[h, 1],[i, 0]\}$. Similarly, for gate $f$, we obtain $[f, 1] \rightarrow[c, 0]$. This process is continued for all gates. Applying transitive law to the implications learned, the complete set of direct implications associated with $g=1$ is:

$$
\operatorname{impl}[g, 1]=\{[g, 1],[c, 0],[e, 1],[f, 1],[h, 1],[i, 0]\}
$$

\section{Indirect Implication}

Although $[h, 1]$ or $[i, 0]$ could not imply anything on node $k$ individually, when combined they can imply $[k, 1]$. This is an indirect implication which is learned through logic simulation. $[k, 1]$ will be added into the implication list for $[g, 1]$, resulting in

$$
\begin{aligned}
& \text { impl }[g, 1]= \\
& \{[g, 1],[c, 0],[e, 1],[f, 1],[h, 1],[i, 0],[k, 1]\}
\end{aligned}
$$

\section{Extended Backward Implication}

Finally, extended backward implications apply to unjustified gates in the implication list. In our example, gate $e$ is an unjustified gate, since the output signal of $e$ is specified as $1([e, 1])$, but none of its inputs (i.e., $a, b$ ) was specified. Thus, $e$ is a candidate for the application of extended backward implication.

To compute extended implications on gate $e$, first, one of the unspecified inputs (gate $a$ ) is set to logic value 1 , we simulate $[a, 1]$ together with all the values in $\operatorname{impl}[g, 1]$. And four implications are found: $\{[d, 0],[j, 0],[p, 0],[q, 0,1]\}$. Next, we simulate $[b, 1]$ together with $\operatorname{impl}[\mathrm{g}, 1]$, we obtain the implications $\{[m, 0],[n, 0],[p, 0][q, 0,1]\}$. Since the implications $[p, 0]$ and $[q, 0,1]$ are common between the two sets obtained from the simulation of $\{[a, 1], i m p l[g, 1]\}$ 
and $\{[b, 1], \operatorname{impl}[g, 1]\}$, they are added into the implication list for $[g, 1]$. Therefore, the complete implication for $[g, 1]$ becomes

$\operatorname{impl}[g, 1]=\{[g, 1],[c, 0],[e, 1],[f, 1],[h, 1],[i, 0]$,

$[k, 1],[p, 0],[q, 0,1]\}$

These implications are stored in a compact implication graph as shown in Figure 2, where each node corresponds to a circuit node assignment [gate=value], each directed edge denotes an implication and the weight on the edge represents the relative time-frame associated with the implication.

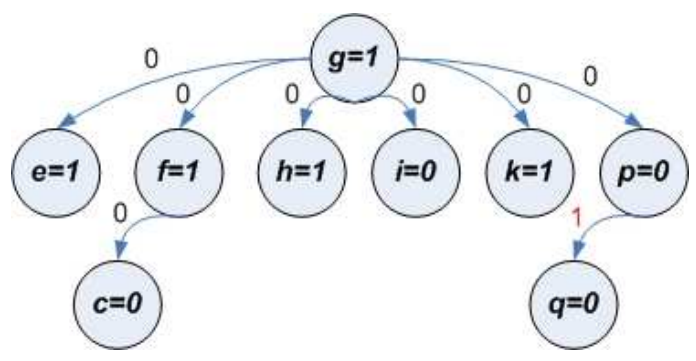

Figure 2: Implication Graph for [g,1]

\subsection{Fault Dominance}

If a fault $\mathbf{f}$ dominates $\mathbf{g}$ in the combinational portion of a circuit, then any test $t$ that detects $\mathbf{g}$ will also detect $\mathbf{f}$ $\left(T_{g} \subseteq T_{f}\right)$ [14]. Knowing this, we can also make the following observation: if $\mathbf{f}$ is untestable, then $\mathbf{g}$ must be untestable as well. Otherwise, there must be at least one test that can detect both faults $\mathbf{g}$ and $\mathbf{f}$. Furthermore, if both faults $\mathbf{f}$ and $\mathbf{g}$ dominate each other, then we say these two faults are equivalent.

\section{Our Approach}

In this section, we describe our proposed method that consists of two phases.

\subsection{Phase 1: Untestable Transition Fault Identification with Static Implications}

In Phase 1, the algorithm similar to $[15,16]$ is used, which identifies a set of untestable stuck-at faults that requires conflicting value assignment for detection in a circuit. However, unlike $[15,16]$, where implications did not span multiple times, our method computes sequential implications quickly without explicit unrolling of the circuit. Unlike FIRES that considers only single-line-conflict scenario, our proposed transition implication engine maximizes the local conflicting assignment in the circuit and extends it to transition fault model.

In $[15,16]$, two sets of faults, set $_{0}$ and set $_{1}$ are computed with respect to a given node $n$ : set $_{i}=$ set of faults that require $[n, i]$ as a necessary condition for excitation or propagation.

Then set $_{0} \cap$ set $_{1}$ are the set of faults that require both $[n, 0]$ and $[n, 1]$ simultaneously for detection, which must then be untestable.

For transition faults, our transition implication engine computes four sets of transition faults, ini_set $_{0}$,

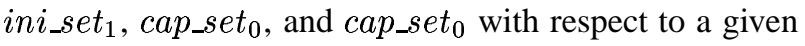
node $n$ :

ini_set $_{i}=$ set of transition faults that require $[n, i,-1]$ as a necessary condition for initialization.

cap_set $_{i}=$ set of transition faults that require $[n, i]$ as a necessary condition for launch or propagation.

We further make the following three definitions:

Definition 1 A transition fault $\ell$ slow-to-rise(slow-tofall) is said to be sequentially uninitializable if there exists no input sequence $T$ such that the targeted line $\ell$ could be initialized to logic 0(1) functionally.

Based on Definition 1, all the transition faults belonging to $i n i_{\_} s e t_{0} \cap i n i_{-}$set $_{1}$ for any given node $n$ would be sequentially uninitializable because they require both $[n, 0,-1]$ and $[n, 1,-1]$ to set the target node to initial value in the immediate preceding time frame.

Definition 2 A transition fault $\ell$ slow-to-rise(slow-tofall) is said to be sequentially uncapturable if there exists no input sequence $T$ such that the stuck-at-O(stuckat-1) fault on line $\ell$ could be sequentially launched and propagated.

Based on Definition 2, all the transition faults belonging to $c a p \_s e t_{0} \cap c a p \_s e t_{1}$ for any given node $n$ would be sequentially uncapturable, because they require both $[n, 0]$ and $[n, 1]$ to launch or propagate the target transition fault.

Definition 3 A transition fault $\ell$ slow-to-rise(slow-tofall) is said to be sequentially constrained if the initialization of $\ell$ in time frame $i-1$ and capture of fault effect on $\ell$ in time frame $i$ require conflicting value assignments.

Thus, the sequentially constrained transition faults are those that are either (1) sequentially initializable but uncapturable in the immediate next time frame, or (2) sequentially capturable but not initializable in the immediate preceding time frame. Note that these three types of untestable transition faults are mutually exclusive. Therefore, the sequentially constrained transition faults for any given node $n$ are $\left(\right.$ ini_set $_{0} \cup$ cap_set $\left._{0}\right) \cap\left(\right.$ ini_set $_{1} \cup$ cap_set $_{1}$ ).

We describe below the High-level description of the implication-based algorithm used in Phase 1. 
1. Construct sequential implication graph through static learning

2. Use both single-line-conflict and maximizing local conflict algorithms to identify the initial set of functionally untestable transition faults, $S 0$.

3. Classify the faults in $S 0$ into three categories based on the definitions given above:

(a) Type 1: Sequentially uninitializable transition faults

(b) Type2: Sequentially uncapturable transition faults

(c) Type 3: Sequentially constrained transition faults

\subsection{Phase 2: Dominated Untestable Transi- tion Faults Identification}

In Phase 2, we define three types of transition fault dominance relationship and describe how they can help identify additional functionally untestable transition faults based on those untestable faults identified in Phase 1. Because the implication-based algorithm in Phase 1 may not be complete, in Phase 2, additional functionally untestable transition faults are identified by finding the dominated fault sets on the previous identified untestable transition faults.

Definition 4 Let $T I_{g}$ and $T I_{f}$ be the sets of all single time-frame vectors $(F F s+P I s)$ that initialize transition faults $\mathbf{g}$ and $\mathbf{f}$, respectively. We say that transition fault $\mathbf{f}$ Initialization-dominates transition fault $\mathbf{g}$ iff $T I_{g} \subseteq T I_{f}$.

Definition 5 Let $T C_{g}$ and $T C_{f}$ be the sets of all single time-frame vectors that capture transition faults $\mathbf{g}$ and f, respectively. We say that transition fault $\mathbf{f}$ Capturedominates transition fault $\mathbf{g}$ iff $T C_{g} \subseteq T C_{f}$.

Definition 6 Let $T I_{g}$ and $T I_{f}$ be the sets of all single time-frame vectors that initialize transition faults $\mathbf{g}$ and $\mathbf{f}$, respectively, in time-frame $t-1$, and let $T C_{g}$ and $T C_{f}$ be the sets of all single time-frame vectors that capture transition faults $\mathbf{g}$ and $\mathbf{f}$, respectively, in time-frame $t$. We say that transition fault $\mathbf{f}$ Constraint-dominates transition fault $\mathbf{g}$ if both of the following two conditions are met:

1. $T I_{g} \subseteq T I_{f}$.

2. $T C_{g} \subseteq T C_{f}$.

We will use the circuit in Figure 3 to illustrate the three types of dominance relationship.

The complete initialization dominance relationship and capture dominance relationship are stored in the two graphs as shown in Figures 4 and 5. In the dominance graph, each node corresponds to a transition fault in the

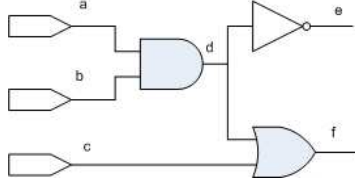

Figure 3: segment of sequential circuit
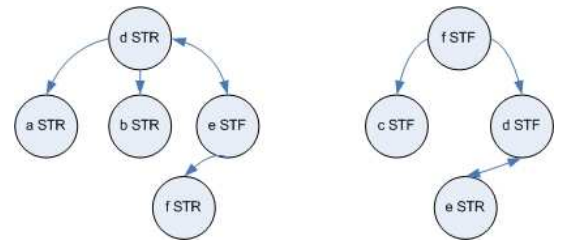

Figure 4: Initialization Dominance Graph

circuit, each directed edge denotes a dominance relationship. These graphs can be derived directly from the structural analysis of the circuit. If we consider Figures 4 and 5 together. We have the following observations:

1. d slow-to-rise dominates a slow-to-rise because both TI and TC for $d$ slow-to-rise are supersets of TI and $\mathrm{TC}$, respectively, for a slow-to-rise.

2. Likewise, $d$ slow-to-rise dominates $b$ slow-to-rise as well as e slow-to-fall. Also, e slow-to-fall dominates d slow-to-rise.

3. However, a slow-to-rise does NOT dominate $d$ slowto-rise, because test vector X0X could initialize $d$ slow-to-rise but not a slow-to-rise.

4. Note that $d$ slow-to-rise and e slow-to-fall dominates each other. Therefore, they are equivalent transition faults.

The dominated untestable faults identification algorithm used in Phased 2 is given as follows:

1. Construct the fault-initialization dominance graph (INI_DOM) and the fault-capture dominance graph (CAP_DOM).

2. SO = initial set of untestable transition faults computed in Phase 1. S1=S2=S3=EMPTY

3. for every identified untestable transition faults $f$ in SO:

(a) case $f \in$ Type1: Search the fault-initialization dominance graph (INI_DOM) to identify dominated untestable faults by $f$ and add them in $(S 1)$

(b) case $f \in$ Type2: Search the fault-capture dominance graph (CAP_DOM) to identify dominated untestable faults by $f$ and add them in $(S 2)$

(c) case $f \in$ Type 3 :

tf set $_{0}=$ untestable transition faults that are initialization-dominated by $f$ 


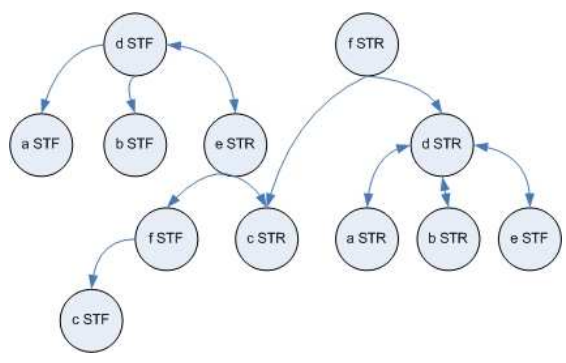

Figure 5: Capture Dominance Graph

$t f$ set $_{1}=$ untestable transition faults that are capture-dominated by $f$

The untestable fault set: $\mathbf{S 3}=$ $\mathbf{S 3} \cup\left(\right.$ tfset $_{\mathbf{0}} \cap$ tfset $\left._{\mathbf{1}}\right)$

4. The final, complete set of functionally untestable transition faults: $\mathbf{S}=\mathbf{S} 0 \cup \mathbf{S} 1 \cup \mathbf{S 2} \cup \mathbf{S 3}$

\section{Experimental Results}

The algorithms explained above were implemented in $\mathrm{C}++$. Experimental data are collected for non-scan ISCAS89 sequential benchmark circuits, on a $2.0 \mathrm{GHz}$ Pentium 4 with $512 \mathrm{MB}$ of memory, running the Linux Operating System. The result of our proposed two-phase procedure on ISCAS 89 benchmark circuits are reported in Table 1, For each circuit, we first give the number of transition faults in the circuit. Next, we show the number of functionally untestable transition faults identified in Phase 1. In column 4-7, we report the number of sequentially uninitializable transition faults, sequentially uncapturable transition faults, sequentially constrained transition faults and total number of functionally untestable transition faults identified by our proposed approach, respectively. Next, column Time reports the corresponding CPU execution time for Phase 2 procedure.

From this table, many additional functionally untestable transition faults have been identified using our approach with very little computational effort. For example, in circuit s386, the implication-only algorithm in Phase 1 identified 195 untestable transition faults, and Phase 2 found 91 additional untestable ones, making a total of 286 faults identified as untestable, in only 0.02 second extra execution time. The number of additional functionally untestable transition faults identified in large sequential circuits (except for s35932) could be significant. For example, in s38417r, the implication-based technique found 6434 functionally untestable transition faults, while the proposed method identified totally 7106 , which is 672 more, in only 12 seconds. For s35932, since the implication-based procedure already identified all the functionally untestable transition faults, no additional faults could be identified by the new technique.
Table 1: Experimental Results using Implication \& Dominance

\begin{tabular}{|c|c|c|c|c|c|c|c|}
\hline \multicolumn{2}{|c|}{ CKT } & \#Functional Untestable Faults Identified & Time \\
\cline { 1 - 5 } Name & \#Faults & Phase1 & \multicolumn{3}{|c|}{ Phase1+Phase2 } & \\
\cline { 4 - 7 } & & & \#UnInit & \#UnCap & \#Constr & \#Total & \\
\hline s298 & 812 & 129 & 8 & 8 & 141 & 157 & 0.01 \\
s344 & 980 & 66 & 11 & 10 & 52 & 73 & 0.01 \\
s386 & 1078 & 195 & 6 & 16 & 264 & 286 & 0.02 \\
s444 & 1180 & 226 & 6 & 22 & 231 & 259 & 0.02 \\
s526 & 1390 & 345 & 0 & 1 & 400 & 401 & 0.03 \\
s641 & 2078 & 66 & 0 & 0 & 88 & 88 & 0.03 \\
s713 & 2206 & 208 & 40 & 81 & 120 & 241 & 0.03 \\
s832 & 2244 & 96 & 0 & 8 & 131 & 139 & 0.03 \\
s953 & 2516 & 81 & 0 & 0 & 109 & 109 & 0.03 \\
s1196 & 3232 & 5 & 0 & 0 & 8 & 8 & 0.04 \\
s1238 & 3254 & 58 & 0 & 50 & 13 & 63 & 0.04 \\
s1423 & 3992 & 387 & 0 & 33 & 463 & 496 & 0.07 \\
s1488 & 4196 & 22 & 0 & 0 & 38 & 38 & 0.06 \\
s1494 & 4196 & 30 & 0 & 11 & 34 & 45 & 0.05 \\
s5378 & 14964 & 3695 & 1090 & 1002 & 1742 & 3834 & 0.70 \\
s9234 & 28174 & 7415 & 284 & 1358 & 6389 & 8031 & 3.14 \\
s13207r & 41758 & 14542 & 2397 & 1255 & 11488 & 15140 & 18.52 \\
s15850r & 49654 & 16402 & 416 & 1190 & 15741 & 17347 & 13.86 \\
s35932 & 96930 & 11255 & 0 & 9536 & 1719 & 11255 & 3.86 \\
s38417r & 116310 & 6434 & 376 & 577 & 6153 & 7106 & 12.09 \\
s38584r & 111578 & 14923 & 1919 & 5975 & 8031 & 15925 & 15.17 \\
\hline \hline Sum & 492722 & 76580 & 6553 & 21133 & 53355 & 81041 & 52.64 \\
\hline
\end{tabular}

Overall, the proposed two-phase approach improve the number of identified functionally untestable transition faults by $5.8 \%$ over the implication-based method in less than 1 minutes totally.

In Table 2, we compare our results with the implication-based method and a ATPG-based approach [6] for some of the large ISCAS89 benchmark circuits. After the first two columns for circuit name and number of transition faults in the circuit, we report the number of untestable transition faults identified by Chen etc. in [6], using a ATPG-based scheme. Then, we give the number of untestable ones identified by implications only and our new technique, respectively. In the last column, we show the percentage improvement our approach has over the ATPG-based method.

For three circuits (s1238, s1488 and s1494) out of the 13 large benchmark circuits, the ATPG-based method [6] performed better. In all the other cases, our proposed technique identified more functionally untestable transition faults than the other two methods. For example, in circuit $\mathrm{s} 38417 \mathrm{r}$, our technique identified 7106 functionally untestable transition faults, which is more than twice of 3075 , the number of untestable transition faults identified in [6]. Overall, our new approach identified $43 \%$ more functionally untestable transition faults than the ATPG- 
Table 2: Comparison With Previous Work

\begin{tabular}{|c|c|c|c|c|c|}
\hline \multicolumn{2}{|c|}{ CKT } & \multicolumn{2}{|c|}{ Func. Unt. faults } & Improvement \\
\cline { 1 - 5 } Name & \#Faults & {$[6]$} & Impl & Ours & $(\%)$ \\
\hline s953 & 2516 & 101 & 81 & $\mathbf{1 0 9}$ & 7.9 \\
s1196 & 3232 & 2 & 5 & $\mathbf{8}$ & 300 \\
s1238 & 3254 & $\mathbf{8 2}$ & 58 & 63 & -23.2 \\
s1423 & 3992 & 273 & 387 & $\mathbf{4 9 6}$ & 81.7 \\
s1488 & 4196 & $\mathbf{2 4 1}$ & 22 & 38 & -84.2 \\
s1494 & 4196 & $\mathbf{2 6 2}$ & 30 & 45 & -82.8 \\
s5378 & 14964 & 1645 & 3695 & $\mathbf{3 8 3 4}$ & 133.1 \\
s9234 & 28174 & 5600 & 7415 & $\mathbf{8 0 3 1}$ & 43.4 \\
s13207r & 41758 & 10637 & 14542 & $\mathbf{1 5 1 4 0}$ & 42.3 \\
s15850r & 49654 & 10259 & 16402 & $\mathbf{1 7 3 4 7}$ & 69.1 \\
s35932 & 96930 & 8903 & $\mathbf{1 1 2 5 5}$ & $\mathbf{1 1 2 5 5}$ & 26.4 \\
s38417r & 116310 & 3075 & 6434 & $\mathbf{7 1 0 6}$ & 131.1 \\
s38584r & 111578 & 14450 & 14923 & $\mathbf{1 5 9 2 5}$ & 10.2 \\
\hline \hline Total & 480754 & 55530 & 75249 & 79397 & 43.0 \\
\hline
\end{tabular}

based method in [6].

\section{Conclusions}

In this paper, we have presented a new approach on identifying functionally untestable transition faults in non-scan sequential circuits. We formulate a new dominance relationship for transition faults and use it to help us identify more untestable transition faults. The proposed technique consists of two phases: first, a large number of functionally untestable transition faults was identified by using the fault-independent logic implications implicitly crossing multiple time-frames and classified into three categories according to the sequential constraint on their initialization and capture requirement. Then, additional functionally untestable transition faults were identified by finding the dominated fault sets on the identified untestable transition faults. The experimental results for ISCAS89 sequential benchmark circuits showed that the proposed two-phase approach identified 5.8\% more functionally untestable faults than implication-based method and $43 \%$ over the ATPG-based approach previously reported.

\section{References}

[1] J. Rearick. Too much Delay Fault Coverage is a Bad Thing. In Proc. of ITC, pages 624-633, 2001.

[2] X. Liu and M. S. Hsiao. Constrained ATPG for Broadside Transition Testing. In Proc. of DFT, pages 175-182, 2003.

[3] D. Brand and V. S. Iyengar. Identification of Redundant Delay Faults. Trans. on CAD, Vol. 13(5):553565, May 1994.
[4] Z. Li, Y. Min, and R. K. Brayton. Efficient Identification of Non-Robustly Untestable Path Delay Faults. In Proc. of ITC, pages 992-997, 1997.

[5] K. Heragu, J. H. Patel, and V. D. Agrawal. Fast Identification of Untestable Delay Faults Using Implication. In Proc. of ICCAD, pages 642-647, 1997.

[6] G. Chen, S. M. Reddy, and I. Pomenranz. Procedures for Identifying Untestable and Redundant Transition Faults in Synchronous Sequential Circuits. In Proc. of ICCD, pages 36-41, 2003.

[7] M. Syal and M. S. Hsiao. Identifying Untestable Transiiton Faults in Latch Based Designs with Multiple Clocks. In Proc. of ITC, 2004.

[8] K.-T. Cheng. Transition Fault Testing for Sequential Circuits. Trans. on CAD, Vol. 12(12), Dec 1993.

[9] J. Rajski and H. Kox. A method to calculate necessary assignments in ATPG. In Proceedings of ITC, pages 25-34, 1990.

[10] S. T. Chakradhar and V. D. Agrawal. A transitive closure algorithm for test generation. Trans. on $C A D$, Vol. 12(7):1015-1028, JULY 1993.

[11] W. Kunz and D. K. Pradhan. Recursive Learning: a new implication technique for efficient solutions to CAD problems-test, verification and optimization. Trans. on CAD, Vol. 13(9):1149-1158, SEPT 1994.

[12] J. Zhao, M. Rudnik, and J. Patel. Static Logic Implication with Application to Fast Redundancy Identification . In Proceedings VLSI Test Symposium, pages 288-293, 1997.

[13] J. Zhao, J. A. Newquist, and J. Patel. A Graph Traversal Based Framework for Sequential Logic Implication with an Application to C-cycle Redundancy Identification. In Proceedings of $V L$ SIDESIGN, pages 163-169, 2001.

[14] M. Abramovici, M. A. Breuer, and A. D. Friedman. Digital Systems Testing and Testable Design. Computer Science Express, 1990.

[15] M. A. Iyer and M. Abramovoci. FIRE: a Fault Independent Combinational Redundancy Algorithm. IEEE Trans. VLSI Systems, 4(2):295-301, June 1996.

[16] M. A. Iyer, D. E. Long, and M. Abramovici. Identifying Sequetially Redundancies without Search. In Proc. of DAC, pages 259-266, 1996. 